\title{
Sistema de información geográfica para el registro de actos delictivos como soporte de las comisarías usando el API de ArcGis
}

\section{Geographic information system for the registration of criminal acts to support police stations using the ArcGis API}

\author{
Pablo Alejandro Jiménez Paz ${ }^{1}$, Katherin Vanessa Rodríguez Zevallos ${ }^{2}$ y Frank Edmundo Escobedo \\ Bailón ${ }^{3}$
}

${ }^{1}$ Universidad Nacional Tecnológica de Lima Sur. Av. Juan Velasco Alvarado, sector 2 grupo 8, mz. D, lote 6 Villa El Salvador, Lima, Perú. Correo electrónico: alejandrojmnz16@ gmail.com. ORCID: https://orcid.org/00000003-4251-6083

${ }^{2}$ Universidad San Ignacio de Loyola. Pasaje Ayancocha 143 - Huánuco, Huánuco, Perú. Correo electrónico: vane251089@ hotmail.com. ORCID: https://orcid.org/0000-0001-8350-720X

${ }^{3}$ Universidad Nacional Tecnológica de Lima Sur. Calle Carlos Alayza y Roel 2392 Dpto. 104 - Lince, Lima, Perú. Correo electrónico: fescobedo@untels.edu.pe. ORCID: https://orcid.org/0000-0002-2058-0976

\section{Resumen}

La inseguridad ciudadana es uno de los problemas más grandes del mundo, en búsqueda de una solución para abordar este problema, nos topamos con que un SIG es una herramienta que vale la pena evaluar para una posible solución. Como objetivo de este estudio, buscamos evaluar la viabilidad de utilizar un SIG para registrar actos delictivos apoyado en las comisarías, para el desarrollo de este proyecto proponemos como marco de desarrollo SCRUM y para la metodología de recojo de información tomaremos artículos, tesis, periódicos y estadísticas externas. A lo largo de algunas investigaciones, podremos hacer distintas comparativas sobre elementos para nuestro software, esto va desde la efectividad que se llega a tener a utilizarlo para mejorar la seguridad ciudadana, las distintas perspectivas que puede tomar los softwares similares (población en tiempo real, población en períodos de tiempo, entidades del estado), hasta la mejor manera de conseguir un mejor costo beneficio para el desarrollo evaluando las diferencias entre los softwares. Por último, logramos concluir que existe un alto grado de efectividad por parte de los SIG, además de que posibilita nuevos métodos de prevención para la mejora de la seguridad ciudadana.

Palabras clave: SIG, crimen, ArcGis, API.

\begin{abstract}
Citizen insecurity is one of the biggest problems in the world, in search of a solution to address this problem, we found that a GIS is a tool worth evaluating for a possible solution. As an objective of this study, we seek to evaluate the feasibility of using a GIS to record criminal acts supported by police stations, for the development of this project we propose SCRUM as a development framework and for the methodology of data collection we will take articles, theses, newspapers and external statistics. Throughout some research, we will be able to make different comparisons about elements for our software, this goes from the effectiveness of using it to improve citizen security, the different perspectives that similar software can take (real time population, time period population, state entities), to the best way to get a better cost benefit for the development by evaluating the differences between the software. Finally, we were able to conclude that there is a high degree of effectiveness on the part of the GIS, in addition to the fact that it makes possible new methods of prevention for the improvement of citizen security.
\end{abstract}

Keywords: GIS, crime, ArcGis, API. 
UNIVERSIDAD NACIONAL DE UCAYALI

Revista de Investigación Universitaria

Versión electrónica $2664-8423$

ARTICULO DE REVISIÓN
Vol. $11 \mathrm{~N}^{\circ} 1$, pp. $540-548$, enero/junio 2021

Recibido 18/02/2021

Aceptado 16/06/2021

Publicado 30/06/2021

\section{Introducción}

Unos de los más grandes problemas de la actualidad son los crímenes (en esta palabra podemos incluir asesinatos, drogas, violaciones, asaltos, entre otros).

Gallup, una agencia de análisis y asesoría estadounidense muestra en una evaluación a 142 países que el Perú se encuentra en el top 10 de países más inseguros, solo siendo superados por Venezuela y México. (Gestión, 2019)

Las instituciones que se encargan de enfrentar la criminalidad en el Perú son las comisarías, pero solo con una mirada al estado de la seguridad ciudadana actual, podemos notar que la cantidad de efectivos policiales disponibles no cubren todas las zonas que presentan actos delincuenciales.

Tomando en cuenta este problema, se propone crear una aplicación web que ubique los puntos de delitos en un área específica y con ellos pueda reportar las áreas más peligrosas y los delitos que se cometen en tales áreas. Con toda esta información se busca posibilitar la reubicación de efectivos policiales en las áreas más afectadas, mejorando así la efectividad de la gestión de recursos.

Para conseguir este objetivo, utilizaremos un sistema de información geográfica, una herramienta que nos ayuda a tratar la información, para poder organizarla y hacerla de fácil entendimiento y gestión. (Hernández, 2016)

\section{Metodología}

El marco de trabajo recomendado para usar en este proyecto es la metodología SCRUM, el motivo de su preferencia es su agilidad al trabajar el desarrollo del software, además de involucrar activamente al usuario asegurándose de cumplir con los requerimientos funcionales planteados, gracias a estos factores asegura una rápida reacción a cambios inesperados. (Schwaber \& Sutherland, 2020)

Otro de las ventajas comparativas del SCRUM es la poca documentación que maneja, ya que se centra en el desarrollo funcional del producto. (Schwaber \& Sutherland, 2020)

El SCRUM como todo marco de trabajo está conformado por un equipo, los miembros del equipo son:

- Product Owner: A quien definimos como el cliente del proyecto.

- Development Team: El equipo que desarrollará el proyecto.

- Scrum Master: El encargado y responsable de ejecutar la metodología adecuadamente. (Schwaber \& Sutherland, 2020)

Para realizar el scrum, usamos algunas herramientas a las que llamaremos artefactos, 
estos serán los siguientes:

- Product Backlog: Requisitos funcionales para desarrollar un producto, define el contenido, el orden y la prioridad de cada uno de estos.

- Sprint Backlog: Subconjunto de requisitos que se implementan en un sprint (fase interna del período de desarrollo).

- Increment: Elementos completados del sprint backlog sumado a los elementos completados de los sprint anteriores. (Schwaber \& Sutherland, 2020)

Por último, las fases que consideramos en la metodología SCRUM son las siguientes:

- Sprint: Tiene un período de duración de menos de 4 semanas y es el tiempo en el que se desarrolla un entregable funcional.

- Sprint Planning Meeting: Se planifican las actividades al realizar el sprint, tiene una duración máxima de 8 horas.

- Daily Scrum: Reunión de duración máxima de 15 minutos donde se plantean las actividades diarias.

- Sprint Review: Revisan las actividades realizadas en el sprint anterior y su impacto en el Producto Backlog.

- Sprint Retrospective: Analiza el comportamiento y la relación de los miembros de equipo, busca potencias las buenas conductas hasta la siguiente fase. (Schwaber \& Sutherland, 2020)
En cuanto al método de recolección de información para este artículo, se realizó una investigación basada en artículos, tesis, publicaciones de periódicos y estadísticas recopiladas por organismos externos.

\section{Resultados y discusión}

\section{Materiales para el desarrollo}

Para poder realizar el proyecto, se recomienda usar los siguientes materiales:

\section{Aplicación web}

Tipo de aplicación especial que usa cliente/servidor, en este tipo se estandariza la relación cliente/servidor y servidor/protocolo. (Luján, 2001)

El protocolo usado para esto es el protocolo HTTP que forma parte de los protocolos que existen dentro de Transmission Control Protocol/Internet Protocol (TCP/IP) que utiliza internet. Este protocolo nos permite conectar sistemas heterogéneos, facilitando así el intercambio entre ordenadores distintos. (Luján, 2001)

\section{JavaScript}

Desarrollado por Brendan Eich entre los años 1995 y 1996, se define como un lenguaje no compilado que inicialmente se concentra en el lado del cliente, utilizando validaciones $\mathrm{y}$ trabajando con imágenes en páginas web, 
UNIVERSIDAD NACIONAL DE UCAYALI

Revista de Investigación Universitaria

Versión electrónica $2664-8423$

ARTICULO DE REVISIÓN
Vol. $11 \mathrm{~N}^{\circ} 1$, pp. $540-548$, enero/junio 2021

Recibido 18/02/2021

Aceptado 16/06/2021

Publicado 30/06/2021 además de mejorar la interactividad y retroalimentación para el usuario. (Suehring, 2013)

Con la llegada de ECMAScript, un estándar que formaliza las prácticas en este lenguaje, JavaScript encontró un auge en su uso, pues permitió mejorar su compatibilidad con distintos navegadores, tales como Internet Explorer, Chrome, Firefox, Opera, Safari y otros, este estándar esta siempre cambiando y agregando mejoras para el desarrollo. (Suehring, 2013)

Si hablamos de JavaScript, no podemos dejar de lado al Document Object Model (DOM) un estándar desarrollado por la World Wide Web Consortium (W3C), este es una interfaz de programación para documentos HTML y XML que facilita que se pueda representar un documento de manera estructurada y define la manera en que los programas pueden acceder, para poder modificar estructura, estilo y contenido. El DOM no cuenta como lenguaje de programación, sin embargo, sirve como medio para darle noción al lenguaje JavaScript de una página web y de los elementos con los que está relacionada. Aunque al principio estuvo herméticamente enlazado con JavaScript, en la actualidad está diseñado para ser independiente a cualquier lenguaje de programación particular y lo hace disponible para estos desde una simple API. (MDN Web Docs, 2019)
Entre las ventajas de usar JavaScript, podemos rescatar las siguientes:

- Útil para desarrollar páginas web dinámicas.

- Consume poca memoria.

- Lenguaje sencillo y liviano.

- Buen manejo de la validación de datos del lado del cliente.

- Compatibilidad con navegadores antiguos y la mayoría de los modernos.

- No necesita un compilador para ser ejecutado.

- Se ejecuta del lado del cliente, por lo que no pide recursos de más al servidor. (Peña \& Cambisaca, 2015)

Como desventaja principal tenemos que el código visible y puede ser leído por cualquiera, por más que existen diversos estándares de seguridad para restringir ejecución de código en los navegadores, aún es posible ejecutar códigos que dañen, roben o destruyan la información del cliente. (Peña \& Cambisaca, 2015)

\section{Angular}

Marco estructural para aplicaciones web dinámicas. (Calmet, 2014)

Permite usar HTML como lenguaje de plantillas para entender la sintaxis de HTML para expresar componentes de la aplicación de 
UNIVERSIDAD NACIONAL DE UCAYALI

Revista de Investigación Universitaria

Versión electrónica $2664-8423$

ARTICULO DE REVISIÓN
Vol. $11 N^{\circ} 1$, pp. $540-548$, enero/junio 2021

Recibido 18/02/2021

Aceptado 16/06/2021

Publicado 30/06/2021 manera más clara y concisa. (Calmet, 2014)

Angular ayuda a la eliminación de código al usar el enlace de datos entre este y la inyección de dependencias, como todo esto sucede en el navegador, Angular es la tecnología ideal para el servidor. (Calmet, 2014)

También crea bloques HTML que minimizan la concordancia entre el documento HTML y las necesidades de una aplicación, para enseñar su sintaxis usada al navegador utiliza construcciones llamadas directivas. (Calmet, 2014)

Ejemplos de directivas podrían ser:

- Enlaces de datos como $\{\{\}\}$

- Estructuras para repetir u ocultar fragmentos del DOM.

- Formas y validaciones de formularios.

- Agrupar HTML en documentos utilizables.

- Coloca código para elementos del DOM. (Calmet, 2014)

La estructura que usamos en Angular es MVC del lado del cliente, el código está escrito en JavaScript y nos ayuda a mostrar modernas páginas web siguiendo un estilo AJAX. (Calmet, 2014)

La versión actual de Angular es la versión 11, pero las versiones 9 y 10 aún cuentan con soporte.

\section{Sistema de Información Geográfica}

Marco de trabajo que reúne, gestiona y analiza datos recopilados.

Especializado en el análisis de ubicaciones, además de la organización de capas de información para poder visualizarlas, además de contar con soporte para esquemas $3 \mathrm{D}$ de distintos modelos geográficos. (Aeroterra, s.f.)

Nos ayuda a reconocer patrones, relaciones y situaciones, mejorando así nuestra toma de decisiones. (Aeroterra, s.f.)

\section{ArcGis Online}

Una serie de herramientas desarrollada por la empresa Esri, que desarrolla y comercializa software para Sistemas de Información Geográfica, para su uso online, proporciona un API que puede ser invocada por medio de JavaScript para usarla en una aplicación web. (Esri, s.f)

Esta API nos permite utilizar mapas 2D y 3D, además de herramientas como:

- Análisis por parte del cliente.

- Graficar en un mapa.

- Editar ubicaciones y mapas.

- Diseño responsive.

- Operaciones geométricas.

- Búsqueda por código geográfico.

- Rutas e indicaciones. 
- Variedad de capas.

- Datos demográficos.

- Análisis territorios desde el espacio. (Esri, s.f)

Proponemos el uso de ArcGis para la realización del proyecto por su versatilidad al trabajar con datos geográficos.

\section{Estado del arte}

Ramírez (2016) realizó una aplicación en la cual registra zonas inseguras en la ciudad de Valparaíso en Chile, el modo de trabajo de esta aplicación se basa en recolectar estos reportes de la ciudadanía misma, esto es una opción muy viable para poder recolectar grandes cantidades de datos, sin embargo, se incurre en la posibilidad de registrar actos falsos dañando así la integridad de nuestra información.

Del mismo modo que la investigación anterior Garzón (2018) nos propone realizar un sistema que ubique por georreferenciación los delitos, y los compare con la distribución de fuerzas policíacas, para poder determinar una correcta redistribución del personal. Una distinción sobre el trabajo anterior, sería el método de recolección de datos, pues este se centra en recolectar datos en períodos de tiempo utilizando encuestas y otros medios de análisis, mientras que nuestro trabajo previo utiliza la aplicación para recibir las actualizaciones en tiempo real, una ventaja del proyecto actual es que se mejora la fiabilidad de los datos al poder filtrarlos de mejor manera.

Desde el otro lado López y su grupo de investigadores (2020) en Piura, desarrollan una aplicación móvil que consume los datos de la comisaría de Castilla para identificar zonas peligrosas en dicho lugar, esta aplicación comparte la información con la población para así poder mejorar la seguridad ciudadana, como inciso a observar podemos señalar que la fiabilidad de los datos mejora considerablemente al consumir los registros de un establecimiento reconocido.

Claros (2018) presenta el uso de QGIS (herramienta similar a ArcGis) para determinar la criminología que existe en las fiestas que se realizan en Magdalena, España. Lo más resaltante en la investigación es que se sale del estándar de solo investigar los actos criminales, sino que también estudia las conductas para poder predecir delitos, todo esto gracias a datos recolectados por llamadas a la policía en las fiestas. Para finalidad de este trabajo, podemos apoyarnos en el uso de este software y hacer comparaciones de este con ArcGis.

Evaluaremos también el artículo de Villanueva (2018) que nos muestra un SIG desarrollado en interfaz web que nos brinda un claro ejemplo de su implementación, sus ventajas, datos sobre el tiempo de respuesta y lo más importante que se rescata es la comparación entre los SIG de software de pago y los de software libre. 
UNIVERSIDAD NACIONAL DE UCAYALI

Revista de Investigación Universitaria

Versión electrónica $2664-8423$

ARTICULO DE REVISIÓN
Vol. $11 \mathrm{~N}^{\circ} 1$, pp. $540-548$, enero/junio 2021

Recibido 18/02/2021

Aceptado 16/06/2021

Publicado 30/06/2021
Siguiendo una visión distinta Opazo (2014) nos muestra cómo aplicar un SIG para monitorear los pastizales en la región de Magallanes, Chile. Para cumplir este objetivo se utiliza MODIS, una herramienta que proporciona imágenes satelitales constantes de la superficie de la tierra, esto brinda una ventaja comparativa sobre otros proyectos realizados anteriormente pues a diferencia de estos se pueden actualizar los resultados de manera periódica mejorando así la precisión con la que se ven los pastizales y mejorando la toma de decisiones en el proceso, todo esto mostrado con herramientas de desarrollo web como HTML y JavaScript.

Continuando con los sistemas web, llegamos al artículo aplicado por Pantoja et al. (2014) que utiliza Google Maps y ArcGIS para servidores creando una SIG a modo de plantilla que muestra la potencia de los sistemas geo colaborativos, esto involucra alertas, mensajes, mapas, métodos de participación ciudadana e instituciones entre otros, tomando siempre como objetivo el utilizar la herramienta para identificar fenómenos geolocalizados.

Por último, veremos el trabajo realizado por Espinoza (2013) que evalúa la implementación de un SIG para identificar los pasivos hidrocarburos utilizando imágenes satelitales recogidas con Google Earth, ArcGIS para el posicionamiento y procesamiento de los datos georreferenciados, y Visual Basic para el control y visualización de los reportes generados por estos datos. Como punto a resaltar, en este proyecto apreciamos un uso completamente distinto de la aplicación ArcGIS que nos proporciona un argumento sólido sobre la viabilidad de su aplicación en distintas áreas.

\section{Conclusiones}

Un SIG de información geográfica nos sirve para poder ordenar información de manera gráfica en un mapa y con esta poder realizar predicciones, mejoras de gestión y muchas otras maneras que nos permiten utilizar esa información.

Entonces al registrar los actos delictivos en un SIG, es posible que los datos recolectados nos muestren un panorama más acertado de la afluencia de los actos delictivos en distintas zonas, además de conocer su ubicación, también es posible conocer el tipo de acto que se comete. Con estos datos almacenados, podemos no solo mejorar la gestión del personal de una comisaría, sino también realizar programas de apoyo en secciones de la población que tengan mayor afluencia de cierto delito, por ejemplo, el crear mejores zonas de recreación en lugares donde se acentúe la drogadicción, charlas sobre la importancia familiar en lugares que se encuentre violencia familiar, y muchos otros programas de mejora posibles ante estos actos; así no solo podríamos actuar cuando suceda un crimen, sino también 
UNIVERSIDAD NACIONAL DE UCAYALI

Revista de Investigación Universitaria

Versión electrónica $2664-8423$

ARTICULO DE REVISIÓN
Vol. $11 \mathrm{~N}^{\circ} 1$, pp. $540-548$, enero/junio 2021

Recibido 18/02/2021

Aceptado 16/06/2021

Publicado 30/06/2021 prevenir que sucedan muchos más.

Por último, podemos discernir que el uso de un software libre sobre uno de paga para utilizar un SIG supone de más trabajo, pero a la larga es mucho más modificable y genera mejor rentabilidad.

\section{Referencias bibliográficas}

Aeroterra (s.f.). ¿Qué es SIG? https://www.aeroterra.com/es-ar/que-esgis/introducción

Calmet, J. F. (2014). Sistema informático web de trámite documentario para la UGEL de Zarumilla - Tumbes utilizando los frameworks AngularJS y Spring MVC. [Tesis de Titulación, Universidad Privada Antenor Orrego]. http://repositorio.upao.edu.pe/handle/up aorep/642

Claros, A. (2018). Uso de datos estadísticos y herramienta QGIS. Estudio estadístico de la criminalidad en las fiestas de Magdalena. [Tesis de Titulación, Universidad Jaime I]. http://hdl.handle.net/10234/177072

Espinoza, L. L. (2013). Aplicación de un sistema de información geográfica para la identificación de pasivos ambientales del subsector hidrocarburos en el Perú para el año 2013. [Tesis de Titulación, Universidad Nacional Federico Villa Real].

http://repositorio.unfv.edu.pe/handle/U $\mathrm{NFV} / 2823$

Esri. (s. f). ArcGIS Online. https://www.esri.com/enus/arcgis/products/arcgisonline/overview

Garzón, I. J. et al. (2018). Desarrollo del modelamiento del sistema de gestión de seguridad y convivencia ciudadana en el municipio de Cáqueza-Cundinamarca a través de ArcGIS. Universidad Libre. http://dx.doi.org/10.21017/rimci.2018.v 5.n10.a48

Gestión. (21 de noviembre de 2019). Perú está en el top 10 de países más inseguros del mundo. https://gestion.pe/economia/peru-en-eltop-10-de-paises-mas- inseguros-detodo-el-mundo-noticia/

Hernández, J. J. (2016). Análisis espacial de índices y percepciones de criminalidad en Colombia. [Tesis de Maestría, UNIGIS].

https://issuu.com/unigis_latina/docs/her nandez

López, A. F. et al. (2020). Aplicación Móvil para acceso a información de zonas peligrosas de robos en el distrito de Castilla - Piura, Perú. [Tesis de Titulación, Universidad Nacional de Piura]. http://repositorio.unp.edu.pe/handle/20. $500.12676 / 2295$

Luján, S. (2001). Programación en internet: Clientes Web. Editorial Club Universitario.

MDN Web Docs (2019). ¿Qué es el DOM?. https://developer.mozilla.org/es/docs/Re ferencia_DOM_de_Gecko/Introducci\% $\mathrm{C} 3 \% \mathrm{~B} 3 \mathrm{n}$

Opazo, S. et al. (2014). Desarrollo de una plataforma web sig para el monitoreo dinámico de pastizales en Magallania. Universidad de Magallanes. http://dx.doi.org/10.4067/S0718686X2014000200003

Pantoja, A. et al. (2014). Integración de servicios de mapas de Google Maps y ArcGIS for Server en la plataforma web GeoCiudadano. UD y la Geomática. https://revistas.udistrital.edu.co/index.p hp/UDGeo/article/view/7912/10948

Peña, J. F. \& Cambisaca, M. G. (2015). Análisis comparativo entre los frameworks JavaScript MVC, AngularJS y EmberJS para el desarrollo de aplicaciones Web. Caso Práctico: "Sistema de control de botiquín veterinario para el MAGAP, Morona Santiago". [Tesis de Titulación, Universidad Técnica Federico de Santa 
UNIVERSIDAD NACIONAL DE UCAYALI

Revista de Investigación Universitaria

Versión electrónica $2664-8423$

ARTICULO DE REVISIÓN
Vol. $11 \mathrm{~N}^{\circ} 1$, pp. $540-548$, enero/junio 2021

Recibido 18/02/2021

Aceptado 16/06/2021

Publicado 30/06/2021
María].

http://dspace.espoch.edu.ec/handle/12 $3456789 / 4583$

Ramírez, M. J. (2016). Geolocalización de eventos delictivos a través de una aplicación móvil social y comunitaria. [Tesis de Titulación, Escuela Superior Politécnica de Chimborazo]. https://repositorio.usm.cl/handle/11673/ 13613

Schwaber, K. \& Sutherland, J. (2020). The Scrum
Guide. https://www.scrumguides.org/docs/scru mguide/v2020/2020-Scrum-GuideUS.pdf

Suehring, S. (2013). JavaScript Step by Step, Third Edition. nSight, Inc.

Villanueva, J. A. (2018). Diseño y desarrollo de un sistema de información geográfica con interfaz web. [Tesis de Titulación, Universidad de Sevilla]. https://hdl.handle.net/11441/83461 